\title{
Children's Dental Diseases and Disabilities
}

\section{Sohaila Ashiq*}

Children's Dental Diseases and Disabilities, General Dentist and Dental Surgeon, Cairo University, Egypt

*Corresponding Author: Sohaila Ashiq, Children's Dental Diseases and Disabilities, General Dentist and Dental Surgeon, Cairo University, Egypt.

Received: October 31, 2019; Published: November 08, 2019

DOI: $10.31080 /$ ASDS.2019.03.0696

A guideline for

- How to treat young patients who suffer from disabilities?

- How to medicate them?

- Medically compromised children.

- Importance of not calling them "Kids with Special needs" and respecting their dignity.

A Disabled child is a child that suffers from mental, physical, medical, or social condition that prevents them from reaching their full potential as human beings due to their condition. Let us think of their suffering and learn how to love them and encourage them to reach their full potential as decent human beings and respect them with dignity.

A periodontium is a connective tissue organ covered by epithelium that attaches the teeth to the bones of the jaws and provides a continually adapting apparatus for support of teeth during function

It is made up of

1. Gingiva

- Papillary portion

- Marginal portion

- Attached gingiva

2. Periodontal ligament (PDL)

3. Alveolar bone

4. Cementum

In Children, there is a higher ratio of them suffering of Gingivitis rather than Periodontitis.

Types of Disabilities and Diseases affecting Children
1. Mental Retardation
2. Down's Syndrome
3. Cerebral Palsy

4. Epilepsy

5. Visual Impairment and Hearing Impairment

6. Heart Diseases

7. Blood Diseases (Hemophilia + Leukemia)

8. Endocrine Disorders

9. Respiratory Diseases

Classification of Gingival diseases in children Simple Gingivitis
a) Eruption Gingivitis,
b) Poor Oral hygiene Gingivitis,
c) Allergy + Gingival inflammation.

Acute Gingivitis

a) Herpes simplex virus infection (HSV1).

b) Recurrent Aphthous ulcers (Canker sore).

c) Acute necrotizing ulcerative gingivitis. (Vincent's infection).

d) Acute candidiasis/ Acute bacterial infections.

Chronic Non-Specific Gingivitis

Gingival diseases by systemic factors
a) Gingival diseases associated with Endocrine system.
b) Gingival Lesions of genetic origin.
c) Gingival overgrowth Phenytoin induced.
d) Gingivitis Ascorbic acid deficiency.

Difference between children's periodontium and adult periodontium

1- Kids have a greater Sulcus depth (1.4 to $2.1 \mathrm{~mm}$ ).

2- Thicker and rounder Free gingiva.

3- Flaccid and retractable Marginal gingiva.

4- Greater width of Attached gingiva.

5- Wide periodontal space. 


\section{Alveolar bone}

- Less calcified,

- More vascular,

- Few thick Trabeculae,

- Thin Lamina Dura

- $\quad$ Flattened Interdental crests.

\section{Assessment}

1- $\quad$ Assess degree of disability.

2- Dental treatment depends on level of retardation/disability.

3- Proper psychological approach:

a. Getting to know the patient reduces their anxiety.

b. You take the time to familiarize them with the clinic and instruments used.

c. Speak slowly and try to use simple and easy terms for them to apprehend.

d. Short and early appointments are preferred

e. Presence of the parents might help, this differs from one case to another.

f. Always use a rubber dam.

Diagnosing + Assessment of a child patient suffering from a disability

\section{Mental Retardation}

This term applies to people whose intellect is below average in comparison to "normal" people.

Degree of mental retardation

a) Mild (IQ: 62-52); special requirements for dental care is $\mathrm{ttt}$ as normal whether sedation or analgesia.

b) Moderate (IQ: 51-36); will require mild to moderate sedation, positive verbal reinforcements.

c) General anesthesia will be required only if severe generalized decay is present. Severe/Profound (IQ: 35 and below); same as above.

- To each and every disease, one must make sure that the medications prescribed won't infuse allergy, are safe to use for kids, pregnant women and their fetuses and most importantly don't contraindicate other medicines used for their disabilities/diseases.

- Also do keep in mind that the type of anesthetics used for the Dental treatments depends on whether the case needs General Anesthesia or a Local one.

Premedication such as:

a) Meprobamate: Used to decrease involuntary movements

b) Chlorpromazine: Used to relieve anxiety c) Librium: Decrease psychic tension and muscle spasms.

- Make Sure that for Visual and Hearing impaired kids are always reassured and the Dentist must establish a trust worthy line of communication and familiarity with the kids prior to treatment.

- A prophylactic antibiotic therapy is always advised if anyone is suffering from Cardiac defects, such as;

a) Amoxicillin is a standard general prophylaxis administered to children with a dosage of $50 \mathrm{mg} / \mathrm{kg}$ orally, 1 hour before the procedure.

b) Ampicillin if the patient is unable to take oral medication, administered $50 \mathrm{gm} / \mathrm{kg}$ IM or IV within 30 mins before the procedure.

c) Clindamycin for those who have allergies from penicillin, $20 \mathrm{mg} / \mathrm{kg}$ orally or IV administered 1 hour/30 mins before the procedure respectively.

- For patients suffering from blood diseases, make sure to avoid drugs that interfere with platelets function as Aspirin and salicylates.

- For patients suffering from Leukemia, make sure to apply when necessary;

a) Obtundents for pain such as Orabase with benzocaine.

b) Topical application of bovine thrombin or Avitence + an oral adhesive for protection.

c) Fungal infections are treated by Nystatin (oral suspension 100,000 units/ml, for 48 hours till lesions disappear.

d) Herpetic lesions are treated by Acyclovir capsules or IV infusion every 8 hours for 7-10 days.

- For patients with respiratory diseases, if an attack occurs during treatment you need to stop immediately, remove any obstacle in the passage of airway and keep it patent, supply with an inhaler. If no improvement occurs, you need to give $0.01 \mathrm{mg} / \mathrm{kg}$ of $1 / 1000$ Epinephrine as a bronchodilator. Corticosteroids must be available and general anesthetic administered treatment is prohibited (hypoxia/lung collapse).

- Metronidazole is a bactericidal, used to treat Gingivitis, along with a proper oral hygiene routine is also administered, vitamin C supplement. Nystatin, a $1 \mathrm{ml}$ suspension/ drops for treatment of oral candidiasis.

- Clindamycin for pericoronitis. Chlorohexidine as a mouth wash.

Volume 3 Issue 12 December 2019

(C) All rights are reserved by Sohaila Ashiq. 